\title{
Radio Tomographic Imaging and Tracking of Stationary and Moving People via Kernel Distance
}

\author{
Yang Zhao \\ Sensor and Signal Analytics \\ Lab, GE Global Research \\ One Research Circle \\ Niskayuna, NY, USA \\ yang.zhao@ge.com
}

\author{
Neal Patwari \\ Department of Electrical and \\ Computer Engineering, \\ University of Utah \\ Salt Lake City, Utah, USA \\ npatwari@ece.utah.edu
}

\author{
Jeff M. Phillips, Suresh \\ Venkatasubramanian \\ School of Computing, \\ University of Utah \\ Salt Lake City, Utah, USA \\ jeffp,suresh@cs.utah.edu
}

\begin{abstract}
Network radio frequency (RF) environment sensing (NRES) systems pinpoint and track people in buildings using changes in the signal strength measurements made by a wireless sensor network. It has been shown that such systems can locate people who do not participate in the system by wearing any radio device, even through walls, because of the changes that moving people cause to the static wireless sensor network. However, many such systems cannot locate stationary people. We present and evaluate a system which can locate stationary or moving people, without calibration, by using kernel distance to quantify the difference between two histograms of signal strength measurements. From five experiments, we show that our kernel distance-based radio tomographic localization system performs better than the state-of-the-art NRES systems in different non line-of-sight environments.
\end{abstract}

\section{Categories and Subject Descriptors}

H.4 [Information Systems Applications]: Miscellaneous

\section{General Terms}

Algorithms, Design, Performance

\section{Keywords}

Localization, Tracking, Sensor networks

\section{INTRODUCTION}

Localization of people using wireless sensor networks has significant benefits in elder care, security, and smart facility applications $[3,19,20]$. Standard "radio localization" systems locate a transmitter tag, or allow a receiver to estimate its position $[3,16]$. For these mentioned applications, it is critical to be able to locate all people, regardless of whether they carry a radio device. In this paper, we explore

Permission to make digital or hard copies of all or part of this work for personal or classroom use is granted without fee provided that copies are not made or distributed for profit or commercial advantage and that copies bear this notice and the full citation on the first page. To copy otherwise, to republish, to post on servers or to redistribute to lists, requires prior specific permission and/or a fee.

IPSN'13, April 8-11, 2013, Philadelphia, Pennsylvania, USA.

Copyright 2013 ACM 978-1-4503-1959-1/13/04 ...\$15.00. "network RF environment sensing" (NRES), that is, using a static wireless sensor network to create an image map of the people and objects and thus locate them in an area of interest based on the changes they cause in the radio frequency (RF) environment. An extensive review of reported NRES research can be found in [20]. NRES is also called "devicefree localization" [25], "passive localization" [28], or "sensorless sensing" [26]. Unlike infrared or thermal, RF penetrates non-metal walls, and thus NRES is useful for emergency applications. For example, in a hostage situation, police could deploy wireless devices outside of the building and learn in real time where people are located in the building, information that may save live. NRES systems can also be used in emergency situations to help rescuers like firefighters locate victims.

RF-based imaging and localization for emergency applications has been dominated by ultra-wideband (UWB) radar systems. Companies like Camero [1] have developed sophisticated phased array radar systems that are capable of penetrating walls. However, these UWB systems are expensive and are limited to military use only today. An emerging NRES technique is to monitor the received signal strength (RSS) on links in a deployed static network and to use the changes in RSS to infer the location of the people in the deployment area $[28,29,24]$. As opposed to multistatic UWB radar [4] or MIMO radar [10], RSS-based NRES requires no expensive and sophisticated hardware, and thus can be implemented with standard wireless networks and devices. We focus on such RSS-based systems in this paper.

Although different NRES systems have been reported and tested, existing methods fail in particular situations. A common method is to use the change in mean in RSS on a link to indicate the shadowing from a person obstructing the link [18]. Shadowing-based radio tomographic imaging (RTI) uses changes in link RSS mean values to estimate the shadowing loss field in the area of the wireless sensor network [13, 23, 5, 12]. Shadowing-based RTI works well in line-of-sight (LOS) environments. In cluttered and non-LOS areas, the assumption that RSS will decrease when a person is on the line between transmitter and receiver (the link line) fails. On a non-LOS link, the RSS may increase, decrease, or both, while a person is located on the link line [24], thus shadowing-based RTI fails in non-LOS environments. Variance-based NRES methods use the variance of RSS measurements to locate human motion [29, 24]. These methods perform well even in non-LOS environments because a moving person changes the RSS of links as she crosses through 
them, increasing the RSS variance, even when the change in mean of RSS is close to zero. However, a stationary person does not change the RSS variance, thus variance-based methods cannot locate her.

One contribution of this work is to use kernel distance to quantify the change in RSS distribution caused by a person, rather than the change in mean or variance. Using kernel distance allows us to locate a person who is stationary or moving, and who is in an LOS environment or non-LOS environment. In short, mean and variance are just two aspects of a random variable; a good metric like kernel distance quantifies the changes in mean, variance and other distribution features, in one metric. In this paper, we explore different histogram difference metrics including the Kullback-Leibler divergence (KLD) [7] and the kernel distance [21], and find that the kernel distance performs better than other metrics in NRES. A demonstration abstract has presented the idea of using a difference between two histograms as a method for RTI [32], however no algorithms, analysis, or validation was presented.

In general, kernel distance-based NRES methods require a single empty-room calibration, similar to shadowing-based RTI methods. However, a second main contribution of this work, we show that for our proposed NRES system, an empty-room calibration can be replaced with a "long-term histogram" which is calculated during operation, regardless of the presence or absence of people. By enabling online calibration, we allow the NRES system to operate without any empty-room calibration, and thus be used for emergency applications in which operators do not know a priori whether an area is empty or not. We show that simple filtering of online RSS measurements allows one to keep a long-term histogram in memory without significant computational complexity. This long-term histogram is close enough to the histogram which would have been measured in an emptyroom calibration to perform as well as with empty-room calibration. In fact, in situations in which the environment has changed since the empty-room calibration, the long-term histogram is closer to a true empty-room measurement, and NRES performs better with it than with the offline emptyroom calibration.

To summarize, the contribution of this paper is to provide a complete framework for RSS-based environmental inference, which enables localization of both moving and stationary people in both LOS and non-LOS environments, and which uses online calibration so that the system does not rely on "empty-room" offline calibration. We explore this framework using reported measurement sets and new measurement sets we collected for this purpose. We evaluate imaging, locating and tracking using our framework. The results show that some links' RSS measurements do not change significantly while a person crosses the link line, so using any single link for NRES is unreliable. However, in an $\mathrm{N}$-node wireless sensor network, there is redundancy from the $\mathcal{O}\left(N^{2}\right)$ links in the network, and one can reliably locate people in the environment. We formulate a new NRES method that estimates a map of human presence from kernel distances in the network, which we call kernel distance-based radio tomographic imaging (KRTI). Then a person's location is estimated to be the coordinate of the pixel with the maximum image value. We then test tracking a single per- son in the area using a Kalman filter ${ }^{1}$. Experimental results show that KRTI can locate a moving person more accurately than VRTI [24] and SubVRT [30]. For localization of a stationary person, KRTI also outperforms a sequential Monte Carlo method [25] both in localization accuracy and computational efficiency. Note that if a person stays still at a location for a long time such as several minutes, our online calibration would gradually "treat" the person as part of the environment, and thus the person would disappear from our KRTI images.

The rest of the paper is organized as follows. Section 2 presents related work. Section 3 first introduces two types of RSS histograms and defines two histogram difference metrics, then describes how we use these metrics to image, locate and track a person in a wireless sensor network. Section 4 describes experiments used in this paper, and Section 5 shows the imaging, localization and tracking results. We conclude in Section 6.

\section{RELATED WORK}

Many recent research studies have focused on RSS-based NRES method using measurements from a wireless mesh network [29, 22, 24], due to the fact that RSS measurements are inexpensive and available in almost all wireless devices. However, all reported methods so far are ad hoc and incomplete. For example, [23] proposes an RSS modelbased method - shadowing-based RTI, to locate stationary and moving people in LOS environments. Based on a similar model, [5] proposes methods to simultaneously track people and locate sensor nodes. However, these methods do not perform well in non-LOS indoor environments due to the multipath effects. For non-LOS indoor environments, variance-based methods using different network configurations $[29,24]$ have been proposed to locate human motion. For example, RF sensors are placed on the ceiling of a room to track people using the RSSI dynamic, which is calculated by RSS measurements with and without people moving inside the room [29]. Variance-based RTI deploys RF sensors on top of stands outside a residential house to locate and track people walking inside the house [24]. However, these variance-based methods cannot locate stationary people, since they all use certain forms of RSS variance to locate human motion, and stationary people do not cause much RSS variance. A more recent study [25] uses a sequential Monte Carlo method to locate both stationary and moving people. This method works at both LOS and nonLOS environments, however, it requires too much computational complexity and cannot be easily implemented in real-time. Compared with all above methods, our proposed NRES method is the only real-time method that is capable of imaging and locating both stationary and moving people in both LOS and non-LOS environments.

To be able to locate both stationary and moving people, our method requires a long-term histogram from online RSS measurements. However, the measurements used here are unlabelled, which is different from the training measurements used in fingerprint-based methods [17, 27] and the offline calibration used in shadowing-based RTI methods [23, 5]. Some fingerprint-based methods use histograms of RSS

\footnotetext{
${ }^{1}$ Note that KRTI is capable of imaging the presence of multiple people, however, we focus on formulating localization and tracking of a single person in this paper.
} 
for purposes of NRES [17, 22]. During their training period, RSS histograms are recorded on all links in a network as a person stands in a known position, which becomes a fingerprint for a person being at that location. Fingerprints are recorded as the person moves to each possible position in the environment. During operational localization period, the current RSS histogram is compared to all of the fingerprints, and the person is estimated to be at the position with the closest matching fingerprint. These methods require RSS fingerprints at each possible person location (or each combination of persons' locations in the case of multiple people), thus the training effort in fingerprint-based methods could be extensive for a large area. In contrast, shadowingbased RTI requires a single "empty-room" offline calibration. Although this offline calibration does not involve extensive training, an empty-room area may not be available in the event of an emergency. Our online calibration proposed in this paper requires neither collection of location fingerprints nor empty-room calibration.

Finally, background subtraction [9] and foreground detection [33] provide methods to classify, from unlabelled RSS data, when a link's RSS data corresponds to a period of motion near the link or a period of no motion. Such methods are an important part of future NRES systems which do not require an empty-room calibration period. The perspective of the methods proposed in this paper are complementary. The method in [9] requires a link to experience periods of motion and no motion, although unlabelled, so that the estimation algorithm can determine the distribution of RSS measurements in both cases. Both [9] and [33] model the state of the environment near the link as a binary - one of two states, obstructed by a person or not obstructed. Our kernel distance metric only measures a distance from the long-term "normal" condition, and thus might provide more "soft" information when the effect on the RSS may differ by the type or location of the obstruction, or the RSS in the "not obstructed" state does not simply stay close to one mean value.

\section{METHODS}

In this section, we first describe how we calculate shortterm and long-term RSS histograms, and show human presence could increase the difference between these two histograms. Then we define metrics to measure histogram difference, and we formulate imaging, localization and tracking via histogram difference.

Commercial wireless devices return a discrete-valued RSS value with each received packet. We denote the RSS of the $i$ th packet as $y^{i}$. We assume there is a finite set of possible RSS values, of size $N$. For example, if a device measures RSS in a range from $y_{\min }$ to $y_{\max } \mathrm{dBm}$ and quantization is $1 \mathrm{dBm}$, then $N=y_{\max }-y_{\min }+1$. Without loss of generality, we refer to the RSS integer as a number in the range $0, \ldots, N-1$.

We assume that there is a network with $L$ links, and packets are transmitted repeatedly and regularly on each link, so that RSS measurements can be made.

\subsection{Short-Term and Long-Term Histograms}

In our proposed method, a link is characterized by a histogram $\mathbf{h}$ of its recent RSS measurements. The $k$ th element of vector $\mathbf{h}$, that is, $h_{k}$, is the proportion of time that RSS integer $k$ is measured on the link. At time $n$, we denote this

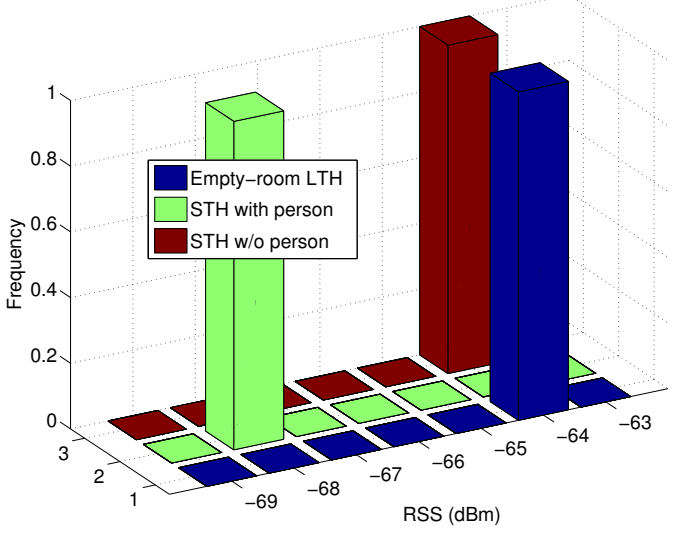

(a) Stationary person effect on STH

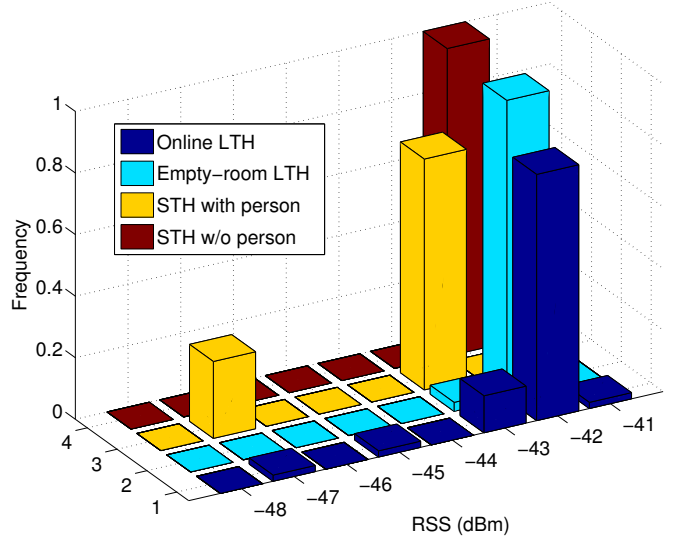

(b) Moving person effect on STH

Figure 1: Long-term histogram (LTH) from offline calibration measurements and short-term histograms (STH) with and without (a) a stationary person; (b) a moving person.

histogram as $\mathbf{h}^{n}$, and calculate it as a filtered version, or weighted average, of RSS measurements:

$$
\mathbf{h}^{n}=\sum_{i} w_{n, i} \mathbf{I}_{y^{i}}
$$

where $y^{i}$ is the RSS at time $i, \mathbf{I}$ is an $N$-length indicator vector, and $w_{n, i}$ is the weight for $\mathbf{I}_{y^{i}}$. The indicator vector $\mathbf{I}_{y^{i}}$ is one in element corresponding to the RSS integer $y^{i}$ and zero in all other elements. Essentially, $\mathbf{I}_{y^{i}}$ is an instantaneous histogram based only on the current measurement, and $\mathbf{h}^{n}$ is a weighted average or filtered version of past instantaneous histograms.

We test two different weighting schemes to compute $\mathbf{h}^{n}$, an offline uniform window, or an exponentially weighted moving average (EWMA). The EWMA scheme has weights,

$$
w_{n, i}=\left\{\begin{array}{lc}
\beta(1-\beta)^{n-i} & i \leq n \\
0 & \text { otherwise }
\end{array},\right.
$$

where $0<\beta<1$ is the forgetting factor. A higher $\beta$ increases the importance of the most recent measurements in the histogram estimate. The EWMA is an infinite impulse 
response (IIR) filter, in which $\mathbf{h}^{n}$ is calculated as,

$$
\mathbf{h}^{n}=(1-\beta) \mathbf{h}^{n-1}+\beta \mathbf{I}_{y^{n}} .
$$

In this way, only the current $\mathrm{RSS}$ value $y^{n}$ and previous histogram $\mathbf{h}^{n-1}$ are necessary to calculate the current histogram. Further, computation of (3) requires $N$ multiplies and a single add. Thus we use the EWMA scheme for all histograms that are computed online, to minimize computational and memory complexity.

A histogram is short-term or long-term based on the chosen weights $w_{n, i}$. For the EWMA filter, the long-term histogram (LTH) would use a lower $\beta$, thus providing more weight to past measurements, than the short-term histogram $(\mathrm{STH})$. In the next sections, we denote the LTH as $\mathbf{q}$ and the STH as p.

The offline uniform window has weight $w_{n, i}$ given as,

$$
w_{n, i}=\left\{\begin{array}{ll}
\frac{1}{T} & 0 \leq i \leq T-1 \\
0 & \text { otherwise }
\end{array} .\right.
$$

If we substitute (4) into (1), we see that the first $T$ RSS values are given equal weight to calculate the histogram. As is clear from the fact that $w_{n, i}$ is not a function of current time $n$, the histogram computed from offline empty-room calibration does not change over time. We use (4) to implement the "empty-room" calibration, that is, we compute the long-term histogram q from (4) when we want to test how our system would have performed if calibrated using data from an initial test period (from 0 to $T$ ) when no person was in the area. The offline uniform window is used purely to compare results when using the proposed online LTH vs. the offline empty-room LTH.

Examples shown in Figure 1 show how the STH and LTH differ for two example links. The empty-room LTH, computed from $T=141$ and the offline uniform window, shows a consistent value of $-64 \mathrm{dBm}$ on the link in Figure 1(a). Two online STHs are shown, both computed with $\beta=0.9$, when a person is present on the link line and when no person is on the link line. With no person present, the STH is nearly identical to the empty-room LTH. When a person stands still on the link line, the STH shows a consistent RSS of $-68 \mathrm{dBm}$. In Figure 1(b), a similar effect is seen - the $\mathrm{STH}$ with no person on the link line is nearly the same as the empty-room LTH. Note also the "STH with person" in this figure is from a time when the person is moving across (rather than standing still on) the link line, and two different RSS values are present in the STH.

Finally, note that Figure 1(b) shows the similarity between the online (EWMA-based) LTH and the offline emptyroom LTH. The online LTH, computed from EWMA with a forgetting factor $\beta=0.05$ does show some non-zero probabilities of other RSS values (e.g., $-41,-43,-45, \ldots$ ), however, the probabilities of these values are very close to zero. It is the fact that these LTHs are very similar which allows us to replace the empty-room calibration, which requires knowing that no person is in the area for a period of time, with an LTH calculated online while people are present and moving in the area. Next, we formalize our discussion of the differences between histograms by defining two histogram difference metrics.

\subsection{Histogram Difference}

There are many ways to measure the difference $D(\mathbf{p}, \mathbf{q})$ between two histograms $\mathbf{p}$ and $\mathbf{q}$. The "earth mover" dis- tance is a popular way of comparing two histograms. However, it involves solving an optimal transportation problem and thus is too computationally expensive for a real-time NRES system. Here, we choose another well known metric, the Kullback-Leibler divergence (KLD) [7]. We also propose to use the kernel distance, which has been recently applied in computational geometry [11].

\subsubsection{Definitions}

The Kullback-Leibler divergence between two histograms $\mathbf{p}$ and $\mathbf{q}$ can be calculated as [7]:

$$
D_{K L}(\mathbf{p}, \mathbf{q})=\sum_{k} p_{k} \log \frac{p_{k}}{\tilde{q}_{k}}
$$

where $\tilde{q}_{k}=\frac{\max \left(\epsilon, q_{k}\right)}{\sum_{k} \max \left(\epsilon, q_{k}\right)}$, and $\epsilon$ is a small number that we use to avoid any divide-by-zero. Note that we investigate the effect of $\epsilon$ later in Section 5.4.

The kernel distance between $\mathbf{p}$ and $\mathbf{q}$ is calculated as $[21]^{2}$ :

$$
D_{K}(\mathbf{p}, \mathbf{q})=\mathbf{p}^{T} \mathbf{K} \mathbf{p}+\mathbf{q}^{T} \mathbf{K} \mathbf{q}-2 \mathbf{p}^{T} \mathbf{K} \mathbf{q}
$$

where $\mathbf{K}$ is an $N$ by $N$ kernel matrix from a 2-D kernel function, and ()$^{T}$ indicates transpose. One commonly used kernel is the Gaussian kernel, defined as:

$$
\mathbf{K}\left(y_{j}, y_{k}\right)=\exp \left(-\frac{\left|y_{j}-y_{k}\right|^{2}}{\sigma_{G}^{2}}\right)
$$

where $y_{j}$ and $y_{k}$ are the $j$ th and $k$ th elements, and $\sigma_{G}^{2}$ is the Gaussian kernel width parameter.

Another common kernel is the Epanechnikov kernel, which is optimal in the sense that it minimizes asymptotic mean integrated squared error [6],

$$
\mathbf{K}\left(y_{j}, y_{k}\right)=\frac{3}{4}\left(1-\frac{\left|y_{j}-y_{k}\right|^{2}}{\sigma_{E}^{2}}\right) I_{\left|y_{j}-y_{k}\right| \leq \sigma_{E}^{2}},
$$

where $I_{a}$ is the indicator function, $I_{a}=1$ where $a$ is true and zero otherwise, and $\sigma_{E}^{2}$ is the Epanechnikov kernel width parameter. Note that the Epanechnikov kernel is not necessarily optimal for KRTI. Both Gaussian and Epanechnikov kernel functions achieve similar performance in KRTI.

\subsubsection{Efficient Implementation}

The computation of (6) has $\mathcal{O}\left(N^{2}\right)$ multiplication and add operations. We show in the following that the kernel distance can be calculated with only $\mathcal{O}(N)$ operations. First, we use the fact that $\mathbf{K}^{\frac{1}{2}}$ is a symmetric matrix $\mathbf{K}^{\frac{1}{2}}=\left(\mathbf{K}^{\frac{1}{2}}\right)^{T}$ to change formulation (6) to the standard Euclidean distance:

$$
\begin{aligned}
D_{K}(\mathbf{p}, \mathbf{q})= & \left(\mathbf{K}^{\frac{1}{2}} \mathbf{p}\right)^{T} \mathbf{K}^{\frac{1}{2}} \mathbf{p}+\left(\mathbf{K}^{\frac{1}{2}} \mathbf{q}\right)^{T} \mathbf{K}^{\frac{1}{2}} \mathbf{q}- \\
& 2\left(\mathbf{K}^{\frac{1}{2}} \mathbf{p}\right)^{T} \mathbf{K}^{\frac{1}{2}} \mathbf{q} \\
= & \left\|\mathbf{K}^{\frac{1}{2}} \mathbf{p}-\mathbf{K}^{\frac{1}{2}} \mathbf{q}\right\|^{2},
\end{aligned}
$$

where $\|\cdot\|$ indicates the Euclidean distance. Letting $\mathbf{u}=$ $K^{\frac{1}{2}} \mathbf{p}, \mathbf{v}=K^{\frac{1}{2}} \mathbf{q}$, we obtain,

$$
D_{K}(\mathbf{p}, \mathbf{q})=\|\mathbf{u}-\mathbf{v}\|^{2} .
$$

Now, consider the online computation of the kernel distance at time $n$, that is, $D_{K}\left(\mathbf{p}^{n}, \mathbf{q}^{n}\right)$, where both LTH and STH are calculated using the EWMA method in (3). Instead of

\footnotetext{
25trickly speaking, definition in (6) is the squared kernel
} distance. We use (6) in KRTI for computation convenience. 


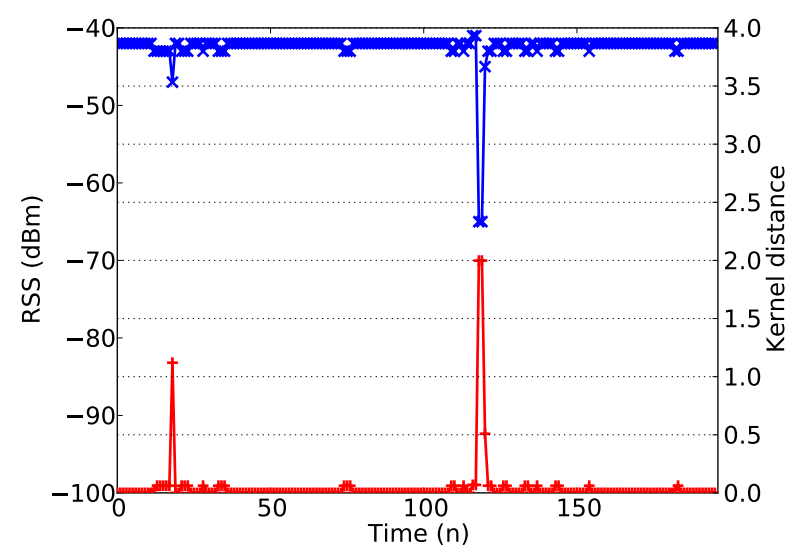

Figure 2: RSS $(\times)$ and kernel distance $(+)$ time series for a link which a person crosses at $n=23$ and $n=120$.

updating $\mathbf{p}^{n}$ and $\mathbf{q}^{n}$ each time $n$, we can reduce computational complexity by instead updating $\mathbf{u}^{n}$ and $\mathbf{v}^{n}$, that is, $\mathbf{u}$ and $\mathbf{v}$ at time $n>0$, using the same EWMA method:

$$
\begin{aligned}
& \mathbf{u}^{n}=\left(1-\beta_{p}\right) \mathbf{u}^{n-1}+\beta_{p} \mathbf{K}^{\frac{1}{2}} \mathbf{I}_{y^{n}} \\
& \mathbf{v}^{n}=\left(1-\beta_{q}\right) \mathbf{v}^{n-1}+\beta_{q} \mathbf{K}^{\frac{1}{2}} \mathbf{I}_{y^{n}}
\end{aligned}
$$

where $y^{n}$ is the RSS at time $n, \beta_{p}$ is the forgetting factor for $\mathbf{u}$, and $\beta_{q}$ is the factor for $\mathbf{v}$. The term $\mathbf{K}^{\frac{1}{2}} \mathbf{I}_{y^{n}}$ is simply the $k$ th column of matrix $\mathbf{K}^{\frac{1}{2}}$, where $k$ is the index of the RSS $y^{n}$ in the histogram, and thus does not require any multiplications. Thus (11) only requires $\mathcal{O}(N)$ multiplies and adds.

Now the current kernel distance at time $n$ is calculated as:

$$
D_{K}\left(\mathbf{u}^{n}, \mathbf{v}^{n}\right)=\left\|\mathbf{u}^{n}-\mathbf{v}^{n}\right\|^{2} .
$$

This formula is identical to $D_{K}\left(\mathbf{p}^{n}, \mathbf{q}^{n}\right)$ except that it requires $\mathcal{O}(N)$, rather than $\mathcal{O}\left(N^{2}\right)$, multiplies and adds. Note that initial values $\mathbf{v}_{0}$ and $\mathbf{u}_{0}$ must be given. We assume that the system has been running prior to $n=0$ and use these initial measurements to initialize $\mathbf{v}_{0}$ and $\mathbf{u}_{0}$.

\subsubsection{Examples}

Consider the example histograms in Figure 1. For Figure $1(\mathrm{a}), D_{K}(\mathbf{p}, \mathbf{q})=0.83$ between the LTH and the STH with a person on the link line, if we use the Epanechnikov kernel with $\sigma_{E}^{2}=30$. Without any people on the link line, $D_{K}\left(\mathbf{p}^{n}, \mathbf{q}^{n}\right)=0$, since the STH is the same as the LTH. For the moving people case in Figure $1(\mathrm{~b}), D_{K}\left(\mathbf{p}^{n}, \mathbf{q}^{n}\right)=1.2$ between the LTH and the STH with people, while $D_{K}\left(\mathbf{p}^{n}, \mathbf{q}^{n}\right)=$ 0.2 if no people near the link. These two examples show that the presence of a stationary and moving person significantly increases the kernel distance.

As another example, we show in Figure 2 both the RSS, $y^{n}$, and kernel distance, $D_{K}\left(\mathbf{p}^{n}, \mathbf{q}^{n}\right)$, for a period of time in which a person crosses the link twice. Kernel distance is very close to zero except when the person crosses the link at $n=23$ and $n=120$, when it exceeds 1.0. Note that $0 \leq D_{K}\left(\mathbf{p}^{n}, \mathbf{q}^{n}\right) \leq 2$. The kernel distance indicates clearly the link crossings by its high value.

\subsection{Kernel Distance-Based Radio Tomographic Imaging}

Let $\mathbf{d}=\left[d_{0}, \ldots, d_{L-1}\right]^{T}$ denote a histogram difference vector with $L$ directional link histogram distances, $d_{l}=$ $D\left(\mathbf{p}_{l}, \mathbf{q}_{l}\right)$. Let $\mathbf{x}=\left[x_{0}, \ldots, x_{M-1}\right]^{T}$ denote an image vector, where $x_{m}$ is a measure of the current presence of a person or object in pixel $m$ that was not typically present in the past. In other words, $x_{m}$ is the "novelty" of human presence in pixel $m$. We assume that $\mathbf{d}$ can be expressed as a linear combination of $\mathbf{x}$, as has been assumed for other RTI systems $[18,13,23,5,12,24]$ :

$$
\mathbf{d}=\mathbf{W} \mathbf{x}+\mathbf{n},
$$

where $\mathbf{n}$ is a vector of measurement noise and model error. We use the elliptical weight model $\mathbf{W}$ given in [23, 24], in which the weight $W_{l, m}$ for pixel $m$ is non-zero only if the pixel center is in an ellipse with foci at the link transmitter and receiver locations.

A radio tomographic image $\hat{\mathbf{x}}$ be estimated from histogram difference measurements $\mathbf{d}$ using:

$$
\hat{\mathbf{x}}=\left(W^{T} C_{\mathbf{n}}^{-1} W+C_{\mathbf{x}}^{-1}\right)^{-1} W^{T} C_{\mathbf{n}}^{-1} \mathbf{d},
$$

where $C_{\mathbf{x}}$ is the covariance matrix of $\mathbf{x}$, and $C_{\mathbf{n}}$ is the covariance matrix of the link measurement noise. Here we use a least squares formulation, which has been shown to outperform the Tikhonov regularization method [31]. The covariance matrix of the link measurement noise, $C_{\mathbf{n}}$, is not generally known here, thus we assume the noise vector has i.i.d. elements. Thus $C_{\mathbf{n}}$ becomes an identity matrix multiplied by $\sigma_{n}^{2}$. We propose to use the following modified least squares formulation:

$$
\hat{\mathbf{x}}=\Pi_{K} \mathbf{d} \quad \text { where } \Pi_{K}=\left(W^{T} W+\sigma_{n}^{2} C_{\mathbf{x}}^{-1}\right)^{-1} W^{T} .
$$

We model the scaled image covariance the same as in [31], where the $(i, j)$ element of $\frac{1}{\sigma_{n}^{2}} C_{\mathbf{x}}$ is given by

$$
\left[\frac{1}{\sigma_{n}^{2}} C_{\mathbf{x}}\right]_{i, j}=\frac{\sigma^{2}}{\delta} \exp \left(-\frac{\left\|\mathbf{r}_{j}-\mathbf{r}_{i}\right\|}{\delta}\right),
$$

where $\sigma^{2}=\sigma_{x}^{2} / \sigma_{n}^{2}$ is the ratio of variance of human presence $\sigma_{x}^{2}$ to the variance of noise $\sigma_{n}^{2}$, which plays the role of regularization, $\delta$ is a correlation distance parameter, $\mathbf{r}_{i}$ and $\mathbf{r}_{j}$ are the center coordinates of the $i$ th and $j$ th pixels, and $\|\cdot\|$ indicates Euclidian distance. From (15) we see the image estimate is the product of $\mathbf{d}$ with a projection matrix $\Pi_{K}$ which can be calculated ahead of time. Thus, the image estimate can be easily calculated in real-time.

In Section 5.4, we compare the performance of KLD and kernel distance for calculation of $\mathbf{d}$ in (15), and show that the kernel distance consistently outperforms the KLD. Thus we generally call our method kernel distance-based radio tomographic imaging, or KRTI. To obtain a good image resolution of human presence, we set the pixel size of KRTI to be 0.3 $\mathrm{m}$ by $0.3 \mathrm{~m}$ in this paper. Then we choose RTI parameters as explained in $[23,24,31]$. We list new parameters and their values used in KRTI in Table 2. Note that we could tune these parameters for a particular experiment to obtain higher localization accuracy, but we use the same parameter values for all experiments to show that KRTI performs well in different environments. We investigate the effects of these parameters on KRTI in Section 5. 


\begin{tabular}{|c|c|c|}
\hline Name & Task & Description \\
\hline Exp.1 & stationary person & calm day through-wall \\
\hline Exp.2 & moving person & calm day through-wall \\
\hline Exp.3 & moving person & windy day with fans \\
\hline Exp.4 & moving person & environment changes \\
\hline Exp.5 & moving person & at a cluttered bookstore \\
\hline
\end{tabular}

Table 1: Experimental datasets.

\subsection{Localization and Tracking}

In this section, we describe how to use the image in (15) to perform localization and tracking, which is the focus of this paper. We assume, for simplifying formulation purpose, that only one person is present in the network. When multiple people are in the area, they can be seen in the KRTI image, however, multi-target localization and tracking is not the focus of this paper.

From the KRTI image estimate $\hat{\mathbf{x}}$, the position of the person is estimated as the center coordinate of the pixel with maximum value. That is,

$$
\hat{\mathbf{z}}=\mathbf{r}_{q} \quad \text { where } \quad q=\underset{p}{\arg \max } \hat{x}_{p}
$$

where $\hat{x}_{p}$ is the $p$ th element of vector $\hat{\mathbf{x}}$ from (15). The localization error of this estimate is defined as: $e_{l o c}=\| \hat{\mathbf{z}}-$ $\mathbf{z} \|$, where $\mathbf{z}$ is the actual position of the person.

To increase accuracy when locating moving people, we apply a Kalman filter to the localization estimates to track people's locations over time. In the state transition model of the Kalman filter, we include both mobile people's location and velocity in the state vector, and the observation inputs of the Kalman filter are the localization estimates. Note that other NRES methods like VRTI [24] is capable of tracking a person even if she stops moving for a while by recording the last location where she was present. However, VRTI cannot image and locate a stationary person that is constantly present at a single location in the network. We evaluate both localization and tracking performance in Section 5 .

\section{EXPERIMENTS}

In this section, we describe experiments that we use in evaluating our new framework. We use TelosB nodes running a network protocol called Spin [2]. At any particular time, only one node is broadcasting while all the other nodes are measuring pairwise RSS. The transmission interval between two nodes is set by the Spin protocol so that three link measurements are recorded each second to match the speed of human motion. For faster human motion, we can increase the transmission frequency at the cost of more power consumption. All nodes are operating on the 26th channel of IEEE 802.15.4 to avoid overlap with WiFi networks. A basestation connected to a laptop listens to the broadcast on that channel and collects RSS from these nodes.

In Experiments 1 and 2, thirty-four radio nodes are deployed outside the living room of a residential house. During the first experiment (Experiment 1), a person is asked to stand motionless at twenty different known locations inside the living room. Experiment 2 is performed with the same settings, but the task is to locate a person walking inside the living room. A person walks around a marked path at a constant speed using a metronome so that the location of the person at any particular time is known. An important

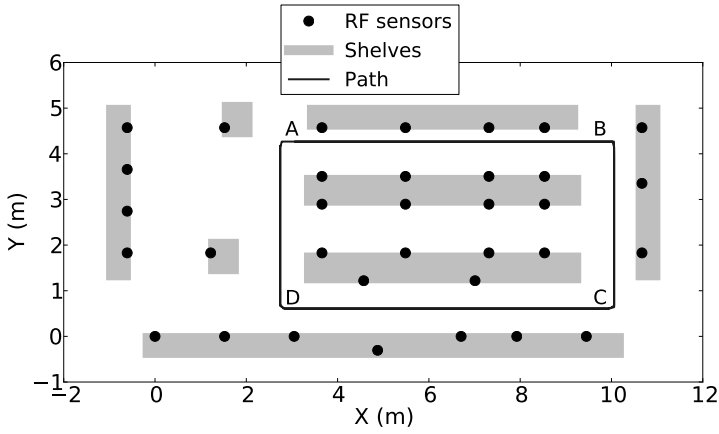

(a) Experiment layout

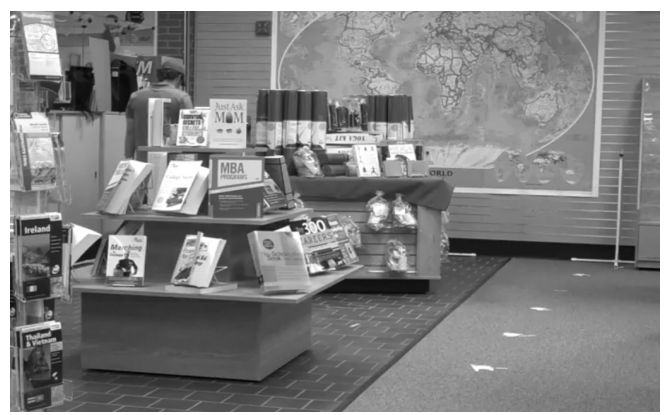

(b) Bookstore environment

Figure 3: Experiment layout and environment of Exp. 5.

fact about Experiment 2 is after recording offline calibration measurements, a node (node ID 32) on the PVC stand was moved to a different location. This system change affects the system performance, which we discuss later. Experiments 1 and 2 are performed and reported by [24]. The third and fourth experiments (Experiment 3 and Experiment 4) are new datasets, which are also through-wall experiments performed at the same residential house with the same hardware and software. Since a recent study [30] demonstrated the degrading effect of wind-induced motion on a variancebased localization system, we choose a windy day and we also place three rotating fans at three locations in the living room to create more motion to increase the background noise for Experiments 3 and 4. Both experiments are performed in the same condition, and both are used to locate a person walking inside the house. The difference is that we observe significant environmental difference between the offline calibration period and the online localization period in Experiment 4. During the offline calibration period, wind blows strongly causing a lot of RSS variations, but it becomes much weaker during online period. We investigate the effect of system and environment changes on the system performance in Section 5.5. The last experiment (Experiment 5) is performed by Wilson et al. [25] in the University of Utah bookstore in an area of about $12 \mathrm{~m}$ by $5 \mathrm{~m}$ with thirty-four nodes deployed on book shelves and display tables. A person walks clockwise around a known path twice from Point A to Point D as shown in the experimental layout Figure 3(a). The bookstore environment is cluttered with shelves, tables and books, as shown in Figure 3(b).

As summarized in Table 1, the first four experiments are all "through-wall" experiments with thirty-four nodes de- 


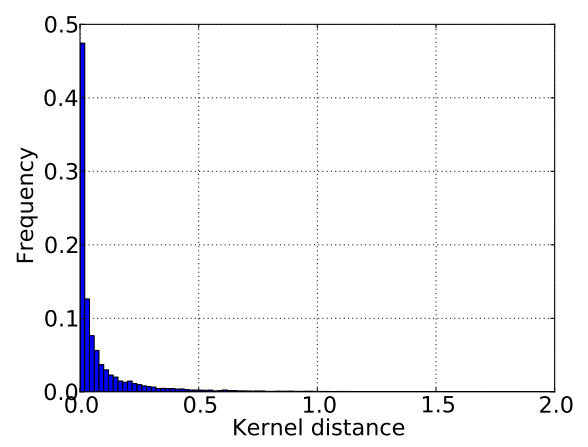

(a) Kernel distance PDF from NPOLL

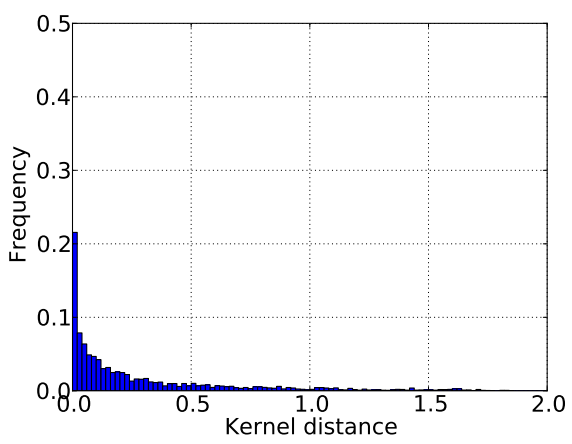

(b) Kernel distance PDF from POLL

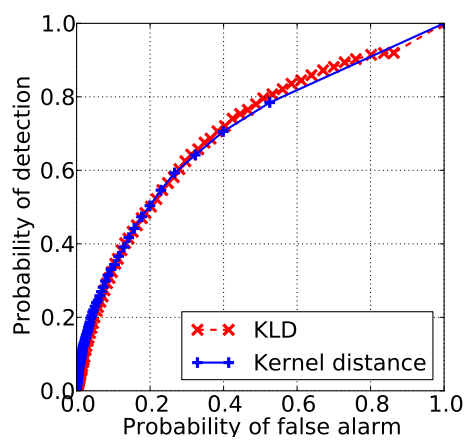

(c) ROC curve

Figure 4: Detection results of using histogram distance to detect a person on link line or not.

ployed outside walls. The directional radio link density for these experiments is about 15 links per $\mathrm{m}^{2}$. For Experiment 5 , the link density is about 19 links per $\mathrm{m}^{2}$. All five experimental environments should be multipath-rich environments.

\section{RESULTS}

In this section, we first evaluate detection via kernel distance, then we show imaging and localization results of a stationary person. After that, we show localization and tracking results of a moving person. Finally, we discuss the performance of using KLD and kernel distance, we also discuss the effect of environment and system change on KRTI performance.

\subsection{Detection of a Person on Link Line}

Before showing the imaging, localization and tracking results, we first test using kernel distance from a single link to detect the presence of a person on a link line. First, we define what we mean by a person being on a link line. We denote the transmitting node and receiving node of link $l$ as $i_{l}$ and $j_{l}$, with coordinates $\mathbf{s}_{i_{l}}$ and $\mathbf{s}_{j_{l}}$, respectively. We denote the person's true location as $\mathbf{z}$. Our definition of "person on the link line" (POLL) is that the person's center coordinate $\mathbf{z}$ is in an ellipse of excess path length $\lambda>0$ with foci at the node locations, that is,

$$
\text { POLL : }\left\|\mathbf{s}_{i_{l}}-\mathbf{z}\right\|+\left\|\mathbf{s}_{j_{l}}-\mathbf{z}\right\|<\left\|\mathbf{s}_{i_{l}}-\mathbf{s}_{j_{l}}\right\|+\lambda .
$$

Note that we use $\lambda=0.06 \mathrm{~m}$ in our results, so that the elliptical area includes only positions very close to the line between the two nodes.

We want to decide between two hypotheses, $H_{0}$ that the NPOLL condition is true, and $H_{1}$ that POLL is true. To avoid making assumptions about the distribution of histogram differences given $H_{0}$ or $H_{1}$, we simply suggest that histogram differences will be higher under $H_{1}$ than under $H_{0}$. Thus, we decide whether we believe NPOLL or POLL is true by comparing the histogram difference to a threshold:

$$
D\left(\mathbf{p}_{l}, \mathbf{q}_{l}\right) \underset{H_{0}}{\stackrel{H_{1}}{\gtrless}} \eta
$$

where $\eta$ is a user-defined detection threshold that is set to be the same for each link, $\mathbf{p}_{l}$ and $\mathbf{q}_{l}$ are the STH and LTH from link $l$, respectively, and $D\left(\mathbf{p}_{l}, \mathbf{q}_{l}\right)$ is calculated from either KLD or kernel distance formulation.

Now, we use data from Experiment 1 and parameters as given in Table 2 to test the detection performance. First, we record all kernel distances during $H_{0}$ (NPOLL). The distribution of $D_{K}\left(\mathbf{p}^{n}, \mathbf{q}^{n}\right)$ given $H_{0}$ is shown in Figure $4(\mathrm{a})$. Approximately half of kernel distances are zero, and the vasty majority are below 0.5. For the data recorded on links where $H_{1}$ (POLL) is true, the kernel distance distribution is shown in Figure 4(b). Now, fewer kernel distances are zero, down to $20 \%$, however, this means that we have no chance of detecting the person standing on the link line for $20 \%$ of links. From the distributions of $D_{K}\left(\mathbf{p}^{n}, \mathbf{q}^{n}\right)$ given $H_{0}$ and $H_{1}$, we calculate the receiver operating characteristic (ROC) curve in Figure 4(c). Even for a probability of false alarm $\left(P_{F A}\right)$ of $40 \%$, the probability of detection is below $80 \%$. Similarly, we test the use of KLD as the difference metric, with the resulting ROC curve shown in Figure 4(c). For low $P_{F A}$, kernel distance has higher detection performance, while for high $P_{F A}$, KLD performs better. The results show the difficulties in detecting human presence using only one link's RSS data. This motivates the use of a network of many links, rather than just a single link, in order to infer the presence and location of people in an area.

\subsection{Imaging and Localization of a Stationary Person}

We now demonstrate that KRTI can not only locate moving people, but also stationary people, a major advantage of KRTI over variance-based methods [24, 29]. In KRTI, we use the EWMA scheme for both long-term and short-term histograms, and the kernel distance, with parameters listed in Table 2. We use measurements from Experiment 1, in which a person stands motionless inside a house, and compare imaging results from KRTI and VRTI [24]. In Figure 5(a), the KRTI image has relatively high pixel values near the true location of the person, and the pixel with maximum value is very close to the true location. Since a stationary human body does not cause much RSS variance, VRTI cannot correctly image the person's location, as shown in Figure 5(b). Note that since EWMA filter is used to update histograms using online measurements, a stationary person staying at one location for several minutes would "fade away" from the KRTI images. However, if a person is 


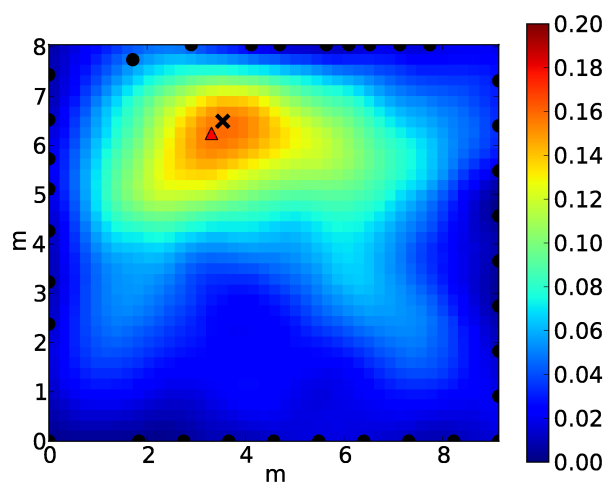

(a) KRTI

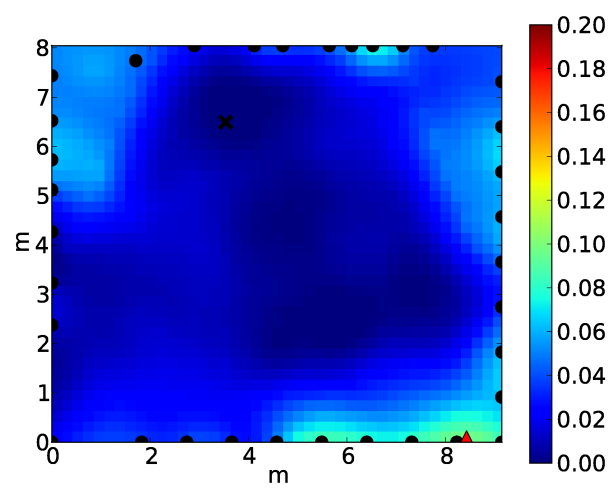

(b) VRTI

Figure 5: Imaging results of a stationary person (true location shown as $\times$ ) from (a) KRTI and (b) VRTI.

in the same location for minutes, we can always record the location where she disappears from the images, and start localization once new motion indicates that she has moved again.

A recent method able to locate a stationary person in a multipath-rich environment is the sequential Monte Carlo (SMC) approach developed by [25]. The method of [25] requires an empty-room (offline) calibration, and is substantially more computational complex than the KRTI method. Further, across experiments, we show that KRTI is more accurate in localization. We run SMC using three hundred particles using data from Experiment 1. In Experiment 1, a person sequentially stands at each of twenty known locations for a constant period $\tau$. At each location we have about fifty KRTI estimates. For these twenty locations, we calculate the overall average error $\bar{e}_{l o c}=\sum_{i=1}^{20}\left\|\hat{\mathbf{z}}_{a}-\mathbf{z}\right\|$, where $\hat{\mathbf{z}}_{a}$ is the average location estimates from KRTI and SMC during period $\tau$. The average location estimates $\hat{\mathbf{z}}_{a}$ from KRTI are shown in Figure 6, in which the line between the average estimate (shown as triangle) and the true location (shown as cross) indicates the estimation error. We see the errors from KRTI are generally below $1 \mathrm{~m}$, more accurate than the results from SMC shown in Figure 10 of [25]. The average error $\bar{e}_{l o c}$ from SMC is $0.83 \mathrm{~m}$, while $\bar{e}_{l o c}$ from KRTI is $0.71 \mathrm{~m}$, a $14.5 \%$ reduction. On the same $2.4 \mathrm{GHz}$

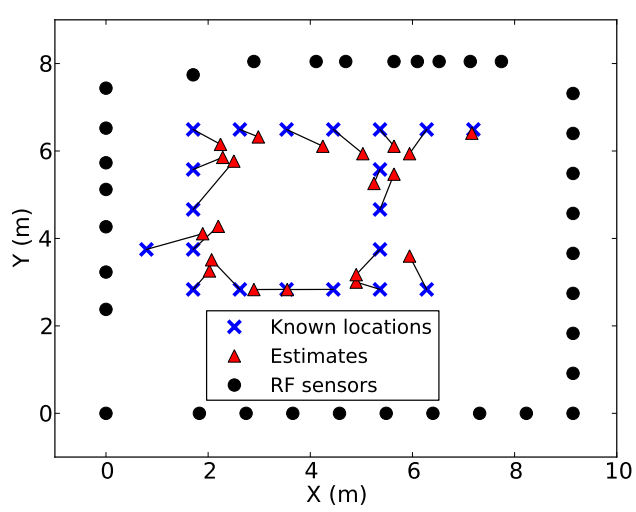

Figure 6: KRTI location estimates of a person standing at twenty locations.

Core 2 Duo processor-based laptop, it takes 0.03 seconds to produce one estimate from our KRTI Python code, while it takes three to four minutes to produce one SMC location estimate. Thus, KRTI outperforms SMC both in accuracy and computational efficiency.

\subsection{Localization and Tracking of a Moving Per- son}

Besides the improvement on imaging and locating stationary people, KRTI also provides better performance for locating moving people. Now we compare KRTI with two variance-based methods, VRTI [24] and SubVRT [30]. We run KRTI, VRTI and SubVRT on data from Experiments 2 5 , and calculate the root mean squared error (RMSE), which is defined as the square root of the average squared localization error. As shown in Table 3, our KRTI can achieve submeter localization accuracy in all experiments. Particularly, for Experiment 3, performed on a windy day, the RMSE from VRTI is $2.1 \mathrm{~m}$, while the RMSE from KRTI is $0.81 \mathrm{~m}$, a $61 \%$ improvement. For Experiment 2, performed on a calm day, SubVRT has a better performance than KRTI $(0.65 \mathrm{~m}$ vs. $0.73 \mathrm{~m}$ RMSE for KRTI). Since SubVRT uses offline empty-room calibration measurements to estimate the noise covariance [30], we expect it to perform particularly well during windy conditions. KRTI does not use such empty-area calibration. However, KRTI significantly outperforms SubVRT, by 30-35\%, in all other experiments. Particularly, in Experiment 4, in which the environment changes between the offline calibration and the online measurements, SubVRT does not perform well. However, KRTI uses online measurements to build the long-term histogram, thus is not significantly affected by offline measurements. The RMSE of KRTI is $0.79 \mathrm{~m}$ in this case, a $31 \%$ improvement on SubVRT. We discuss the effect of environmental changes in more detail in Section 5.5. For Experiment 5, due to the strong multipath environment of the cluttered bookstore (as shown in Figure 3(b)), neither VRTI nor SubVRT perform very well. However, KRTI is particularly robust to non-LOS environments and achieves $0.74 \mathrm{~m}$ RMSE, a similar error as in other experiments. To summarize, KRTI does not just use RSS variance or RSS mean. Instead, it uses histogram difference to include both the effect of a stationary person and a moving person. It is particularly robust to the multi- 


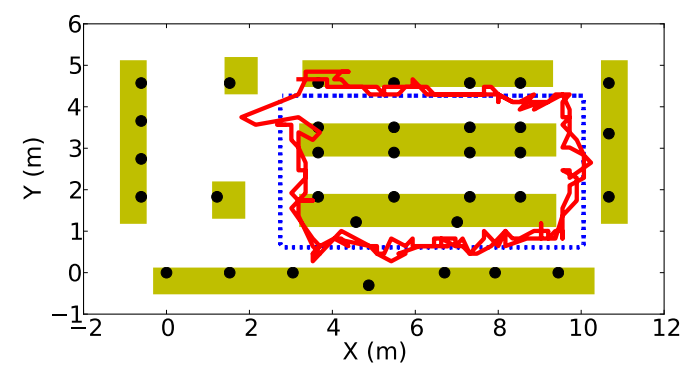

Figure 7: Kalman filter tracking results for Experiment 5 (true path shown as dash line).

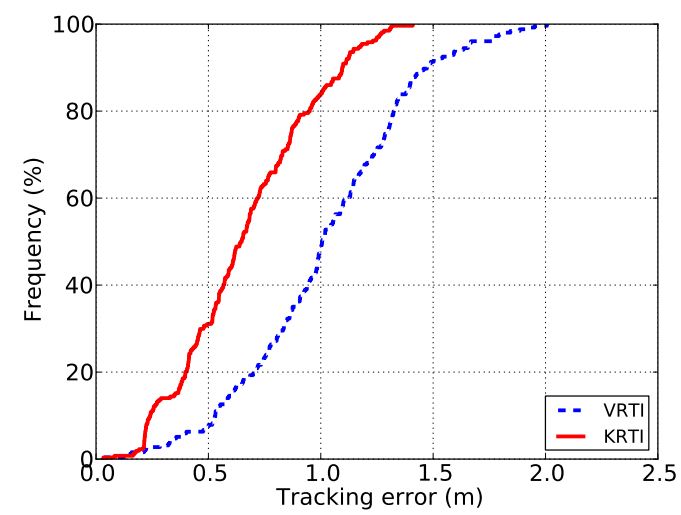

Figure 8: Kalman filter tracking CDFs from VRTI and KRTI for Experiment 5.

path environment, working just as well in strong multipath environments.

Finally, we show the Kalman filter tracking results of Experiment 5 in Figure 7 . We see that tracking results have highest errors when the person is far from the closest radio node. For example, the tracking error is about $1 \mathrm{~m}$ when the person is located at the upper left corner of the path. However, KRTI with a Kalman filter is capable of tracking a person in a large multipath-rich environment with submeter accuracy in general. We also compare the cumulative distribution functions (CDFs) of tracking errors from KRTI and VRTI in Figure 8. For VRTI, 95\% of tracking errors are below $1.7 \mathrm{~m}$, while $95 \%$ of errors from KRTI are below 1.2 $\mathrm{m}$, a $29 \%$ improvement. We also see the median tracking RMSE from VRTI is $1.0 \mathrm{~m}$, while it is $0.6 \mathrm{~m}$ for KRTI, a $40 \%$ improvement.

\subsection{Kernel Distance vs. KLD}

In this section, we compare kernel distance and KullbackLeibler divergence (KLD) as histogram difference metrics in localization. Using an Epanechnikv kernel defined in (8), we test different kernel width parameters $\sigma_{E}^{2}$. Figure 9 shows that KRTI performance is not sensitive to this parameter. RMSEs from Experiments 2 and 3 are both shallow functions of $\sigma_{E}^{2}$, as long as $\sigma_{E}^{2} \geq 10$. A kernel that is too wide tends to smooth the data so much that all measurements look the same. However, a kernel that is too narrow will not smooth the data at all, and as a result is easily affected by noise. The

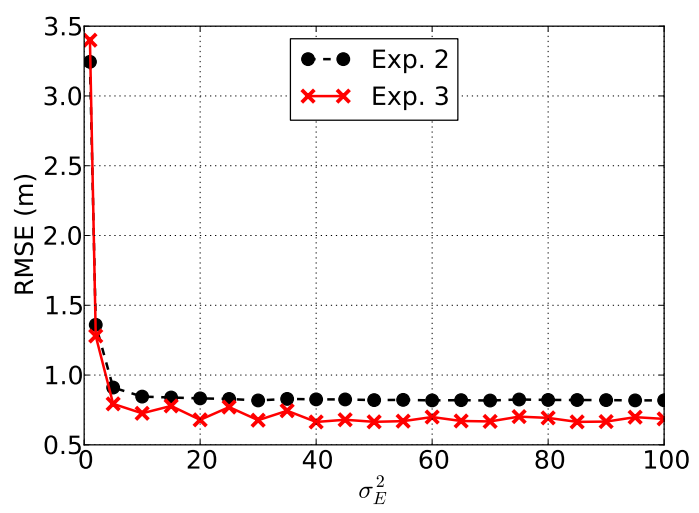

Figure 9: Kernel parameter $\sigma_{E}^{2}$ vs. RMSE from KRTI.

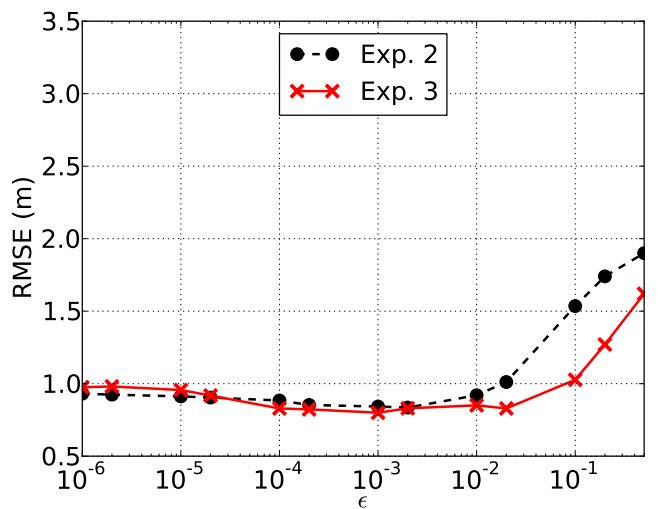

Figure 10: KLD parameter $\epsilon$ vs. RMSE from KRTI using KLD.

kernel width should be chosen so it is roughly proportional to the scale of sensing noise; then a strong signal can still be observed, modest sensing noise will not change the results much, and outliers will tend to be smoothed away.

To calculate KLD, we use parameter $\epsilon$ in (5) to avoid division by zero. As shown in Figure 10, if $\epsilon<0.1$, the localziation RMSE is only mildly sensitive to this parameter. However, from a comparison of Experiments 2 and 3, the RMSEs when using KLD are generally above $0.8 \mathrm{~m}$, while most RMSEs from kernel distance are below $0.8 \mathrm{~m}$. From Figure 9 and Figure 10, we see both histogram difference metrics can achieve submeter localization accuracy, however, kernel distance is less sensitive to its parameter $\sigma_{E}^{2}$, and consistently outperforms KLD in localization accuracy.

\subsection{Effects of Environment and System Changes}

In the above tests, we use the EWMA filter to calculate the online LTH q. We can also use the offline empty-room calibration in order to calculate the LTH. We compare the two in this section.

Note that if the environment changes or sensors change positions after the offline empty-room calibration, the changes diminish system performance. As described in Section 4, the location of a single node is accidentally changed after the offline empty-room calibration period in Experiment 2, prior 


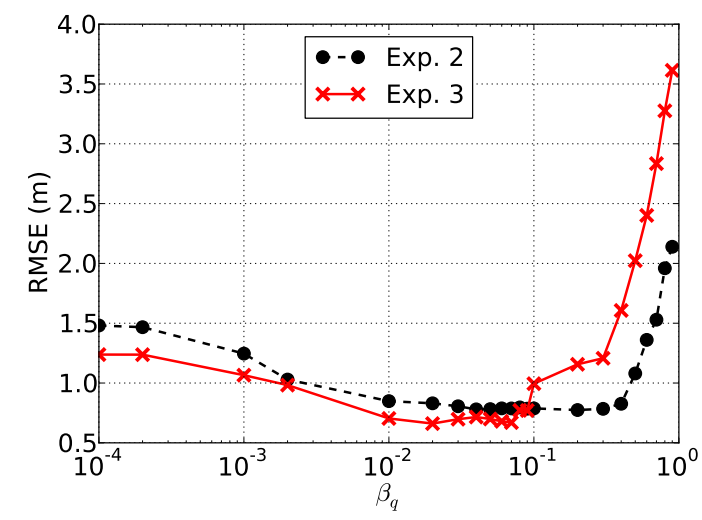

Figure 11: EWMA coefficient $\beta_{q}$ vs. RMSE from KRTI.

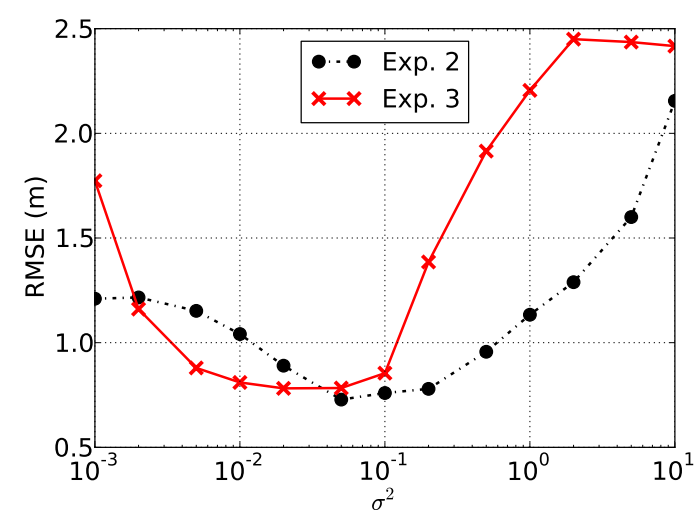

Figure 12: KRTI RMSE vs. $\sigma$.

to the online period. Even if a receiver node moves by only a fraction of its wavelength, its measured RSS values may vary by tens of $\mathrm{dBs}$ as a result of small-scale fading [8]. We apply the offline empty-room LTH in KRTI to generate the image in Figure 14, in which a person is walking and is at the position indicated by the cross. The figure shows two hot spot areas - besides the one close to the true location of the person, there is another one at the lower left corner of the network, close to node 32 indicated by the red circle. A similar false image, not shown, is seen during Experiment 4, in which the environment changes after the offline emptyroom calibration. We avoid this false image problem by using the EWMA for online calculation of the LTH. In our KRTI, we use solely the EWMA filter for online calculation of both long-term and short-term histograms. We do, however, require initialization of the histograms at time zero. In real-time operation, we would simply run the system for a short period to collect sufficient RSS measurements [14] to allow the LTH to "settle" prior to using its results. By using the EWMA filter, our KRTI does not have such false image as shown in Figure 14. To see how EWMA overcomes the effect of position change of a node in Experiment 2, we use the offline calibration measurements as the initialization of LTH, and then use EWMA and online measurements to update LTH in our KRTI. The time series of position estimate error is shown in Figure 15. We see that KRTI estimate

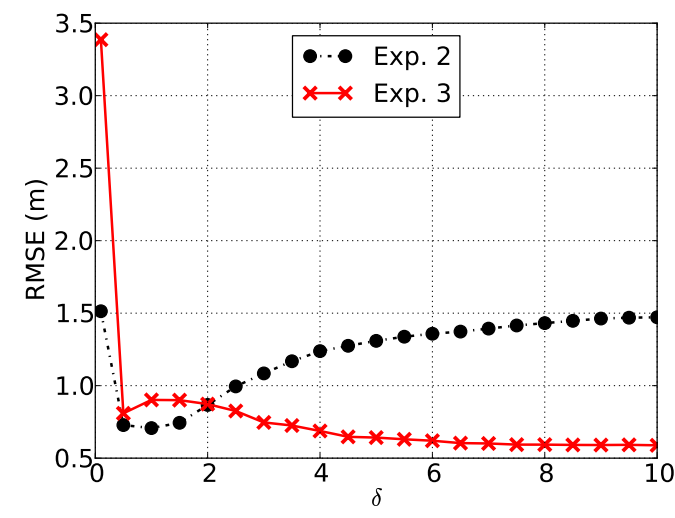

Figure 13: KRTI RMSE vs. $\delta$.

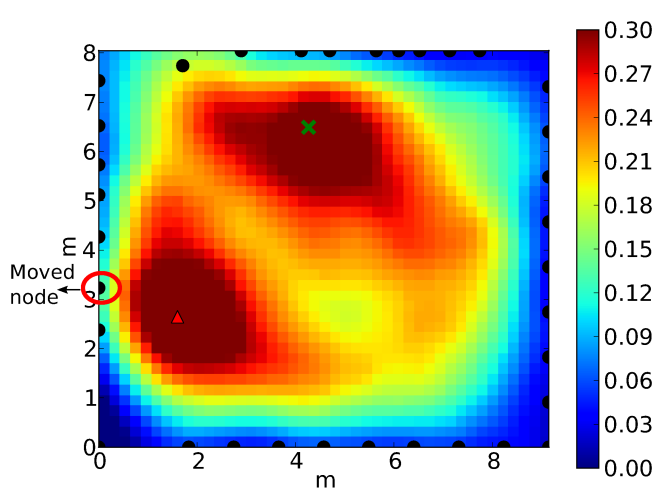

Figure 14: Effect of a moved node on KRTI when using the offline empty-room LTH.

error can be up to $6 \mathrm{~m}$ due to offline LTH affected by the node position change. However, after a few estimates, KRTI errors are all below $2 \mathrm{~m}$. The false hot spot disappears from KRTI images due to the online EWMA update.

We see the relative RMSE performance of empty-room LTH (offline FIR) vs. online LTH (online EWMA) in Table 4 . We see that the online LTH is as good or better than the offline LTH in every case. While the RMSEs are similar in Experiments 3 and 5, the online LTH performs significantly better for Experiments 2 and 4, for which there were either sensor position changes or environmental changes between the empty-room calibration and the online operation, as described earlier. If we control the updating speed appropriately by choosing $\beta_{q}=0.05$, the "online EWMA" method can achieve submeter accuracy for all experiments. Since Experiments 3 and 5 do not have much environment and system change effect, both methods have similar performance.

For KRTI using the online LTH, we test the effect of EWMA forgetting factor $\beta_{q}$. The RMSEs from KRTI with different $\beta_{q}$ values are shown in Figure 11. The RMSEs are very shallow functions of $\beta_{q}$ and are all below $1 \mathrm{~m}$ in the range of 0.01 to 0.1 . If $\beta_{q}$ is below 0.01 , the weight of the latest measurement becomes very small, that is, the update process of the LTH is very slow. If $\beta_{q}=0$, it is equivalent to no update. At the other extreme, if $\beta_{q}$ is too high, i.e., 


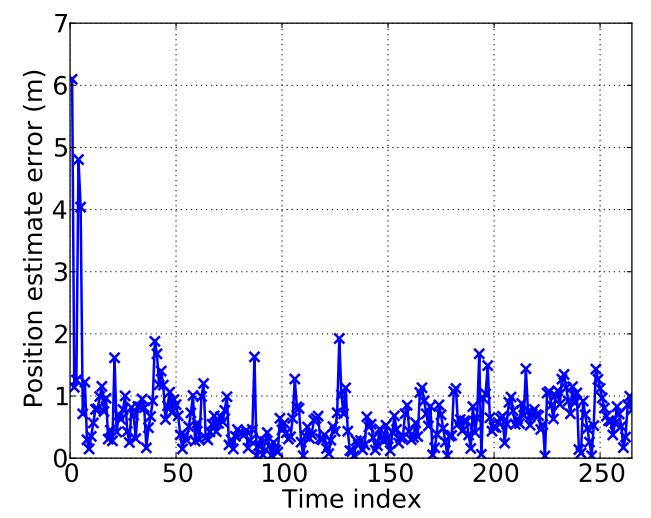

Figure 15: KRTI error time series.

\begin{tabular}{|c|c|c|}
\hline Parameter & Value & Description \\
\hline$\sigma^{2}$ & 0.05 & Regularization parameter \\
\hline$\delta$ & 0.5 & Space parameter $(\mathrm{m})$ \\
\hline$\sigma_{E}^{2}$ & 30 & Epanechnikov kernel width \\
\hline$\epsilon$ & 0.001 & KLD parameter \\
\hline$\beta_{q}$ & 0.05 & EWMA factor for $\mathbf{v}$ \\
\hline$\beta_{p}$ & 0.9 & EWMA factor for $\mathbf{u}$ \\
\hline
\end{tabular}

Table 2: Parameters used in KRTI.

above 0.1 , then the update speed becomes too fast. If $\beta_{q}$ approaches 1 , then almost all previous RSS measurements are removed from the memory. To keep sufficient history measurements in memory and also balance between these two extremes, we choose $\beta_{q}=0.05$ as listed in Table 2 . We also test the effect of EWMA factor $\beta_{p}$ for updating the STH $\mathbf{p}$, we find KRTI performance is best in the range of 0.8 to 1 .

Note that other methods can be used to make an NRES system more robust to the environment and system changes. For example, [30, 31] use subspace method and least squares method to reduce the noise effects due to the environment changes. Work in [15] shows that one can detect when a transmitter or receiver is mis-behaving, and future work might be able to automatically detect failed or moved nodes.

\subsection{Effects of KRTI Parameters}

We have examined the effects of kernel width parameter $\sigma_{E}^{2}$ and EWMA coefficient $\beta_{q}$ on the performance of KRTI in the above two subsections. Now we investigate another two important parameters - the regularization parameter $\sigma^{2}$ and space parameter $\delta$ in our KRTI formulation (16).

Recall that parameter $\sigma^{2}$ is the ratio of human presence variance to noise variance. From (15) and (16), we see that the reciprocal of $\sigma^{2}$ plays the role of regularization parameter as in [24, 23]. To see its effect, we set $\sigma^{2}$ values in a wide range from 0.001 to 10 , fix other parameter values and run KRTI to calculate the RMSEs. Figure 12 shows the RMSEs of KRTI vs. $\sigma^{2}$. We see that when $\sigma^{2}$ is in the range of 0.002 and 0.1, RMSEs from Experiments 2 and 3 are both below $1.3 \mathrm{~m}$, and the RMSE vs. $\sigma^{2}$ curves are shallow. However, when $\sigma^{2}$ is above 0.2 , that is, less regularization is used in the inversion, the RMSEs increase significantly for both experiments due to insufficient smoothing effect from regularization. We also notice that we need a smaller $\sigma^{2}$ value

\begin{tabular}{|c|c|c|c|c|}
\hline RMSE (m) & Exp. 2 & Exp. 3 & Exp. 4 & Exp. 5 \\
\hline VRTI & 0.70 & 2.12 & 1.46 & 1.09 \\
\hline SubVRT & 0.65 & 1.05 & 1.14 & 1.08 \\
\hline KRTI & 0.73 & 0.81 & 0.79 & 0.74 \\
\hline
\end{tabular}

Table 3: RMSEs of locating a moving person.

\begin{tabular}{|c|c|c|c|c|}
\hline RMSE (m) & Exp. 2 & Exp. 3 & Exp. 4 & Exp. 5 \\
\hline Offline FIR & 1.49 & 0.74 & 5.02 & 0.74 \\
\hline Online EWMA & 0.73 & 0.81 & 0.79 & 0.74 \\
\hline
\end{tabular}

Table 4: RMSEs from KRTI using online IIR and offline FIR methods.

for Experiment 3 compared to Experiment 2 to obtain the minimum RMSE. This is due to the fact that Experiment 3 has more noise than Experiment 2. The noise variance of Experiment 3 is greater than that of Experiment 2, thus $\sigma^{2}$ should be set lower for Experiment 3 to keep the variance of human presence a constant. We set $\sigma^{2}=0.05$ for all five experiments.

Another parameter listed in 2 is the correlation distance parameter $\delta$, which controls the spatial size of the exponentially decaying regularization term in (16). While $\sigma^{2}$ controls the intensity of the regularization term, $\delta$ affects both the intensity and the size of the smoothing "blob" (regularization term) in the KRTI images. The RMSEs vs. $\delta$ plot is shown in Figure 13. We see when $\delta=0.1 m$, that is, the size of the smoothing blob is very small, RMSEs of both experiments are higher than $1.5 \mathrm{~m}$. Even if the intensity of the regularization is high, the general smoothing effect is not sufficient due to a small sized blob. As long as the smoothing blob reaches the size of a typical human size of about $0.5 \mathrm{~m}, \mathrm{RMSEs}$ are below $1 \mathrm{~m}$. Increasing $\delta$ beyond $2 \mathrm{~m}$ causes too much smoothing effect for Experiment 2 without much noise, thus RMSE of Experiment 2 increases slowly for $\delta>2$. For Experiment 3 with a lot of noise, a larger blob size averages out additional noise, thus RMSE continues to decrease slowly. For a single person experiment like Experiment 3 , it does not hurt to increase the blob size. However, for tracking more than one person, it would be necessary to limit $\delta$. In this paper, we choose $\delta=0.5$ for all experiments.

\subsection{Discussion}

Compared with other NRES methods, KRTI demonstrates better performance in imaging, localization and tracking. Shadowing-based radio tomographic imaging [23] can locate both stationary and moving people at line-of-sight (LOS) environments, but does not work at multipath-rich environments. Compared with variance-based methods [29, 24, 30], KRTI has the ability of imaging a stationary person as well as a moving person. For tracking a moving person, KRTI also outperforms VRTI and SubVRT. In addition, KRTI

\begin{tabular}{|c|ccc|c|}
\hline Features & RTI[23] & VRTI[24] & SMC[25] & KRTI \\
\hline Through-wall? & No & Yes & Yes & Yes \\
\hline Online calibration? & No & NA & No & Yes \\
\hline Stationary people? & Yes & No & Yes & Yes \\
\hline Real-time? & Yes & Yes & No & Yes \\
\hline
\end{tabular}

Table 5: Features of different NRES methods. 
can use an EWMA filter to update the long-term histogram continuously during an online period, and is more robust to environmental and system changes. The advantage of KRTI over the SMC method [25] is that KRTI does not require any empty-room offline calibration, and performs better both in localization accuracy and computational efficiency. To summarize, KRTI has new properties that other methods do not. We list features of different methods in Table 5. To our knowledge, KRTI is the first NRES method that can locate both stationary and moving people in both LOS and non-LOS environments without any offline calibration.

\section{CONCLUSION}

In this paper, we propose a new NRES framework that uses histogram difference and online calibration to perform network RF sensing of people. Specifically, we propose a kernel distance-based RTI, which uses the kernel distance between a short-term histogram and a long-term histogram to image and locate a moving or stationary person. We explore the framework using three reported measurement sets and two new measurement sets. We evaluate detection, imaging and tracking using our framework. Our experimental results show that KRTI provides robust imaging and tracking capabilities at multipath-rich environments, even though detection from individual links is unreliable. Compared with previous methods, KRTI is the only real-time method that is capable of imaging and locating both stationary and moving people in both LOS and non-LOS environments without any training or empty-room calibration.

\section{REFERENCES}

[1] Camero website. http://www.camero-tech.com.

[2] Sensing and Processing Across Networks (SPAN) Lab, Spin website. http://span.ece.utah.edu/spin.

[3] P. Bahl and V. N. Padmanabhan. RADAR: an in-building RF-based user location and tracking system. In $I E E E$ INFOCOM 2000, volume 2, pages 775-784, 2000.

[4] C. Chang and A. Sahai. Object tracking in a 2D UWB sensor network. In 38th Asilomar Conference on Signals, Systems and Computers, volume 1, pages 1252-1256, Nov. 2004.

[5] X. Chen, A. Edelstein, Y. Li, M. Coates, M. Rabbat, and A. Men. Sequential monte carlo for simultaneous passive device-free tracking and sensor localization using received signal strength measurements. In Proc. ACM/IEEE International Conference on Information Processing in Sensor Networks (IPSN), Chicago, U.S., April 2011.

[6] D. Comaniciu and P. Meer. Mean shift: A robust approach toward feature space analysis. Pattern Analysis and Machine Intelligence, IEEE Transactions on, 24(5):603-619, 2002.

[7] T. Cover and J. A. Thomas. Elements of Information Theory. John Wiley \& Sons, 1991.

[8] G. D. Durgin. Space-Time Wireless Channels. Prentice Hall PTR, 2002.

[9] A. Edelstein and M. Rabbat. Background subtraction for online calibration of baseline rss in rf sensing networks. Technical Report arXiv:1207.1137v1, Arxiv.org, July 2012

[10] A. M. Haimovich, R. S. Blum, and L. J. Cimini. MIMO radar with widely separated antennas. IEEE Signal Processing, 25(1):116-129, Jan. 2008.

[11] S. Joshi, R. V. Kommaraji, J. M. Phillips, and S. Venkatasubramanian. Comparing distributions and shapes using the kernel distance. In Proceedings of the 27th annual ACM symposium on Computational geometry, SoCG '11, pages $47-56,2011$.

[12] O. Kaltiokallio and M. Bocca. Real-time intrusion detection and tracking in indoor environment through distributed RSSI processing. In IEEE 17th International Conference on Embedded and Real-Time Computing Systems and Applications (RTCSA), Toyama, Japan, August 2011.

[13] M. A. Kanso and M. G. Rabbat. Efficient detection and localization of assets in emergency situations. In $3 r d$ Intl.
Symposium on Medical Information $\&$ Communication Technology (ISMICT), Montréal, Québec, Feb. 2009.

[14] J. M. Lucas and M. S. Saccucci. Exponentially weighted moving average control schemes: Properties and enhancements. Technometrics, 32(1):1-12, 1990.

[15] M. Maheshwari, S. A. P.R., A. Banerjee, N. Patwari, and S. K Kasera. Detecting malicious nodes in rss-based localization. In Proceedings of the 2nd IEEE International Workshop on Data Security and Privacy in Wireless Networks (D-SPAN), pages 1-6, June 2011.

[16] G. Mao, B. Fidan, and B. D. O. Anderson. Wireless sensor network localization techniques. Comput. Networks, 51(10):2529-2553, 2007.

[17] M. Moussa and M. Youssef. Smart services for smart environments: Device-free passive detection in real environments. In IEEE PerCom-09, pages 1-6, 2009.

[18] N. Patwari and P. Agrawal. Effects of correlated shadowing: Connectivity, localization, and rf tomography. In $I E E E / A C M$ IPSN'08, April 2008.

[19] N. Patwari, J. Ash, S. Kyperountas, R. M. Moses, A. O. Hero III, and N. S. Correal. Locating the nodes: Cooperative localization in wireless sensor networks. IEEE Signal Process., 22(4):54-69, July 2005.

[20] N. Patwari and J. Wilson. RF sensor networks for device-free localization: Measurements, models and algorithms. Proceedings of the IEEE, 98(11):1961-1973, Nov. 2010.

[21] J. M. Phillips and S. Venkatasubramanian. A gentle introduction to the kernel distance. Technical Report arXiv:1103.1625, Arxiv.org, 2011.

[22] M. Seifeldin and M. Youssef. Nuzzer: A large-scale device-free passive localization system for wireless environments. Technical Report arXiv:0908.0893, Arxiv.org, Aug. 2009.

[23] J. Wilson and N. Patwari. Radio tomographic imaging with wireless networks. IEEE Transactions on Mobile Computing, 9(5):621-632, May 2010.

[24] J. Wilson and N. Patwari. See-through walls: Motion tracking using variance-based radio tomography networks. IEEE Transactions on Mobile Computing, 10(5):612-621, May 2011.

[25] J. Wilson and N. Patwari. A fade level skew-laplace signal strength model for device-free localization with wireless networks. IEEE Transactions on Mobile Computing, 11:947 958, June 2012.

[26] K. Woyach, D. Puccinelli, and M. Haenggi. Sensorless Sensing in Wireless Networks: Implementation and Measurements. In Second International Workshop on Wireless Network Measurement (WiNMee'06), April 2006.

[27] C. Xu, B. Firner, Y. Zhang, R. Howard, J. Li, and X. Lin. Improving RF-based device-free passive localization in cluttered indoor environments through probabilistic classification methods. In Proc. 11th Int. Conf. Information Processing in Sensor Networks, pages 209-220, 2012.

[28] M. Youssef, M. Mah, and A. Agrawala. Challenges: device-free passive localization for wireless environments. In MobiCom '07: ACM Int'l Conf. Mobile Computing and Networking, pages 222-229, 2007.

[29] D. Zhang, J. Ma, Q. Chen, and L. M. Ni. An RF-based system for tracking transceiver-free objects. In IEEE PerCom'O\%, pages $135-144,2007$.

[30] Y. Zhao and N. Patwari. Noise reduction for variance-based device-free localization and tracking. In Proc. of the 8th IEEE Conf. on Sensor, Mesh and Ad Hoc Communications and Networks (SECON'11), June 2011.

[31] Y. Zhao and N. Patwari. Robust estimators for variance-based device-free localization and tracking. Technical Report arXiv:1110.1569v1, Arxiv.org, Oct. 2011.

[32] Y. Zhao and N. Patwari. Histogram distance-based radio tomographic localization. In Proceedings of the 11th international conference on Information Processing in Sensor Networks, IPSN '12, pages 129-130. ACM, 2012.

[33] Y. Zheng and A. Men. Through-wall tracking with radio tomography networks using foreground detection. In Proceedings of the Wireless Communications and Networking Conference (WCNC), pages 3278-3283, Paris, France, April 2012. 